\title{
A Case-Study of Grand-Design Warps in Galactic Disks
}

\author{
Gyula I. G. Józsa ${ }^{1} \dagger$, F. Kenn ${ }^{1}$, U. Klein ${ }^{1}$, and T. A. Oosterloo ${ }^{2}$ \\ ${ }^{1}$ Argelander Institute for Astronomy (AIfA), Univ. Bonn \\ Auf dem Hügel 71, D-53121 Bonn, Germany \\ ${ }^{2}$ Netherlands Foundation for Research in Astronomy \\ Postbus 2, 7990 AA Dwingeloo, The Netherlands
}

\begin{abstract}
We present the results of a case-study of three typical galaxies with integral-shaped warps. We apply a new method to directly fit tilted-ring models to high-quality H I data cubes obtained with the WSRT and report on the features found to be present in the models.
\end{abstract}

Keywords. galaxies: ISM, structure, kinematics and dynamics, individual (NGC 5204, UGC 3580, NGC 2541), methods: data analysis

In order to perform a detailed study of the ubiquituous symmetric ("grand-design") warps in disk galaxies, we selected the galaxies NGC 5204, UGC 3580, and NGC 2541 as suitable candidates with distinctly symmetric H I disks to be observed with the WSRT.

We developed a software tool called TiRiFiC (Tilted-Ring-Fitting-Code) to parametrise the $\mathrm{HI}$ disks of the galaxies in terms of a tilted-ring model. TiRiFiC produces mock observations that are compared directly to input data cubes. The parametrisation is automatically optimised via a $\chi^{2}$ minimisation method (see Józsa 2006).

We confirm Briggs' (1990) well known "rules for the behaviour of warps". Furthermore we observe:

i) At large radii, the orientation of the disk becomes constant again. The galaxy shows a two-disk structure, consisting of an inner disk aligned with the stellar body, and a more or less flat outer disk, the warp being the transition from one orientation to another.

ii) Under the assumption of circular orbits, a change of the rotation velocity occurs at the transition radius (with varying significance).

iii) The H I surface-density drops markedly at the transition radius.

The findings presented here may be typical for evolved, symmetric warps. The structure of the analysed H I disks, viz their outer flat regime, is reminiscent of the galaxy structure found in cosmological simulations by Bailin et al. (2006): after its last merger a galaxy quickly settles into a long-lived intrinsic misalignment; the outer portion of the halo, traced by a tenuous H I disk, is inclined with respect to the inner disk- Dark Matter halo system, which itself is well aligned. While not being the preferred option, the scenario proposed by Shen \& Sellwood (2006), simulating cosmic infall with an external ring of growing (and decreasing) mass, could also be compatible with our findings.

\section{References}

Bailin, J., Kawata, D., Gibson, B. K., et al. 2005, ApJ 627, L17.

Briggs, F. H. 1990, ApJ 352, 15.

Józsa, G. I. G. 2006, PhD thesis, Univ. Bonn.

Shen, J. \& Sellwood, J. A. 2006, MNRAS 370, 2.

$\dagger$ email: gjozsa@astro.uni-bonn.de 